\title{
Numerical Simulations and Conformal Analysis of Growing and Branching Negative Discharge Streamers
}

\author{
Carolynne Montijn, Bernard Meulenbroek, Ute Ebert, and Willem Hundsdorfer
}

\begin{abstract}
The dynamics of an anode-directed streamer can be described by advection-diffusion equations for the charged particles, including a local field-dependent impact ionization term, and coupled to the Poisson equation for the electric field. We present the results of new simulations that use a local uniform grid refinement strategy. Even on very fine grids, provided the electric field is high enough, the streamer appears to branch spontaneously. These results are supported by new analytical solutions based on a moving boundary approximation.
\end{abstract}

Index Terms-Local grid refinement, minimal streamer model, moving boundary approximation, negative streamers.

D ISCHARGE streamers can emerge when a strong potential is applied to a sufficiently large sample of nonconducting matter like a gas. They consist of channels of nonequilibrium plasma that is generated at the channel tip. The rapid propagation of the tip is characterized by local self-induced enhancement of the electric field.

For anode directed streamers, essential properties of the process can be analyzed within a minimal model which contains only two charged species (electrons and positive ions), an impact ionization reaction, and the Poisson equation of electrostatics. The model can be applied to nonattaching gases like nitrogen or argon. In dimensionless units, it is [1]

$$
\begin{aligned}
\partial_{t} \sigma & =\nabla \cdot(\sigma \boldsymbol{E})+D \nabla^{2} \sigma+\sigma|\boldsymbol{E}| e^{-\frac{1}{|\boldsymbol{E}|}} \\
\partial_{t} \rho & =\sigma|\boldsymbol{E}| e^{-\frac{1}{|\boldsymbol{E}|}} \\
\nabla \cdot \boldsymbol{E} & =-\nabla^{2} \phi=\rho-\sigma
\end{aligned}
$$

where $\sigma$ and $\rho$ are the electron or positive ion density, respectively, $D$ is the diffusion coefficient of the electrons, and $\boldsymbol{E}$ and $\phi$ the electric field and potential, respectively. The positive ions can be assumed to be immobile on the short time scales considered here because their mobility is at least two orders of magnitude smaller than that of the electrons [2]. The dimensionless quantities have been defined by scaling the corresponding dimensional quantities with the most natural scales for the length

Manuscript received July 2, 2004; revised January 3, 2005. This work was supported by the Dutch funding agency NWO and by CWI Amsterdam.

C. Montijn, B. Meulenbroek, and W. Hundsdorfer are with the Center for Mathematics and Computer Science (CWI), 1090GB Amsterdam, The Netherlands (e-mail: carolynne.montijn@cwi.nl).

$\mathrm{U}$. Ebert is with the Center for Mathematics and Computer Science (CWI), 1090GB Amsterdam, The Netherlands and also with the Eindhoven University of Technology, $5600 \mathrm{MB}$ Eindhoven, The Netherlands.

Digital Object Identifier 10.1109/TPS.2005.845951 $l_{0}$, time $t_{0}$, electric field $E_{0}$, and charge $q_{0}$. For $N_{2}$ at $300 \mathrm{~K}$, they depend on the pressure $p$ (in bar)

$$
\begin{aligned}
l_{0} & =2.3 \mu \mathrm{m} / p, & t_{0} & =3 \cdot 10^{-12} \mathrm{~s} / p, \\
E_{0} & =200 \mathrm{kVcm}{ }^{-1} p, & q_{0} & =4.7 \cdot 10^{14} e \mathrm{~cm}^{-3} p^{2}
\end{aligned}
$$

where $e$ is the elementary charge.

Up until now, all simulations [2]-[6] performed on this model have been carried out on uniform grids, and some of them [4]-[6] show that, provided the background electric field is high enough, the streamer tends to grow into an unstable state, leading to spontaneous branching. The simulations show very steep ionization fronts around the propagating channel, next to wide inert space where only the Poisson equation in the absence of space charges has to be solved.

We, therefore, have implemented a simulation code with local uniform grid refinement in order to be able to use finer grids [7], to investigate the nature of the instabilities in detail and to be able to deal with larger system sizes. The computational domain is three dimensional with radial symmetry, where $r$ and $z$ are the radial and axial coordinates, respectively. The cathode is planar, situated at $z=0$, and the distance between the electrodes is $L=2000$, corresponding to $4.6 \mathrm{~mm}$. The finest grid size is $1 / 2$. The initial condition is a Gaussian ionization seed at the cathode

$$
\sigma(r, z, t=0)=\rho(r, z, t=0)=\sigma_{0} e^{-\frac{\left(r^{2}+z^{2}\right)}{R_{0}^{2}}}
$$

where $\sigma_{0}$ corresponds to a maximum density of $10^{14} \mathrm{~cm}^{-3}$, and $R_{0}$ is the $1 / e$ radius, corresponding to $25 \mu \mathrm{m}$. The background electric field is set to $\boldsymbol{E}_{b g}=-0.4 \hat{z}, \hat{z}$ being the unit vector in the axial direction, which corresponds to $80 \mathrm{kV} / \mathrm{cm}$. Fig. 1 shows the temporal evolution of the initial ionization seed under these conditions. We used a Neumann boundary condition for the electrons at the cathode, which corresponds to a net flux of electrons into the computational domain. Therefore, the particle densities at the cathode will always grow, and in the figures the densities at the cathode have been cut off in such a way that the front of the channel is well reproduced. The plot of the total charge density at $t=100$ already show pace charge effects. A charged layer appears, strongly affecting the electric field, which becomes relatively low in the body of the streamer and very high at the outside of the streamer front. The ratio of layer thickness over channel radius decreases and approaches an interfacial limit, after which the streamer becomes unstable and branches, as can be seen at $t=500$. 


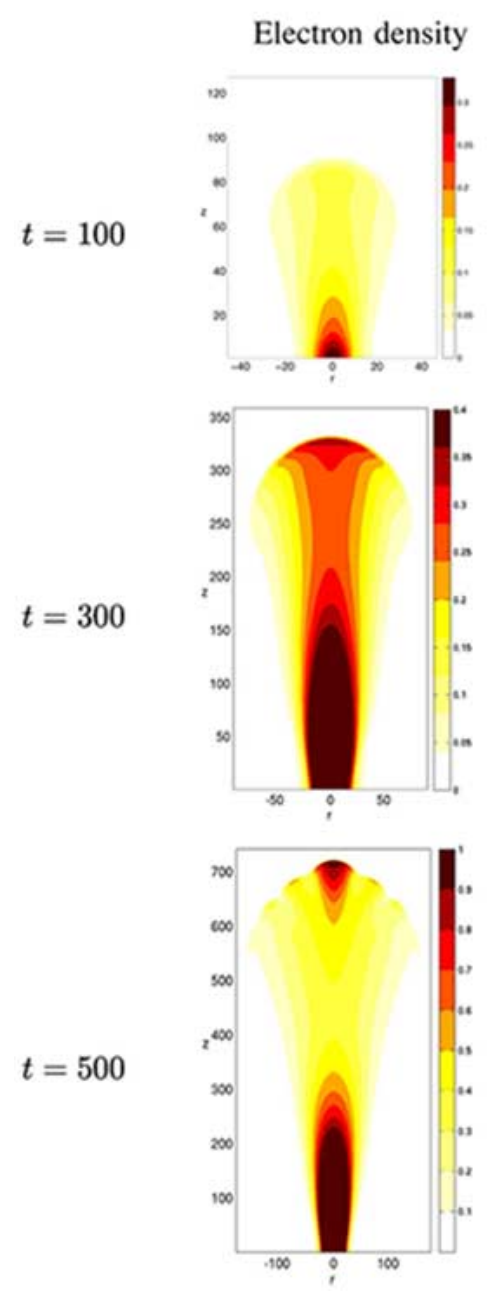

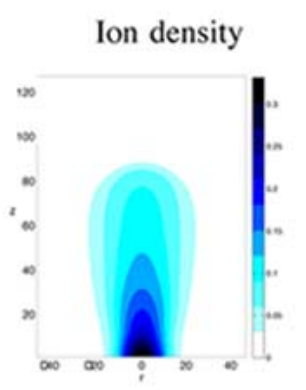
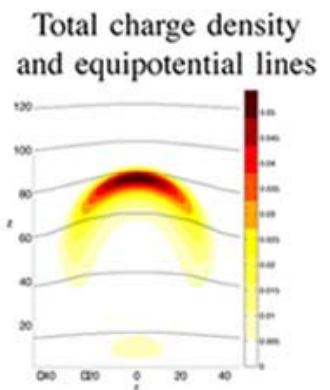

\section{Electric field strength}
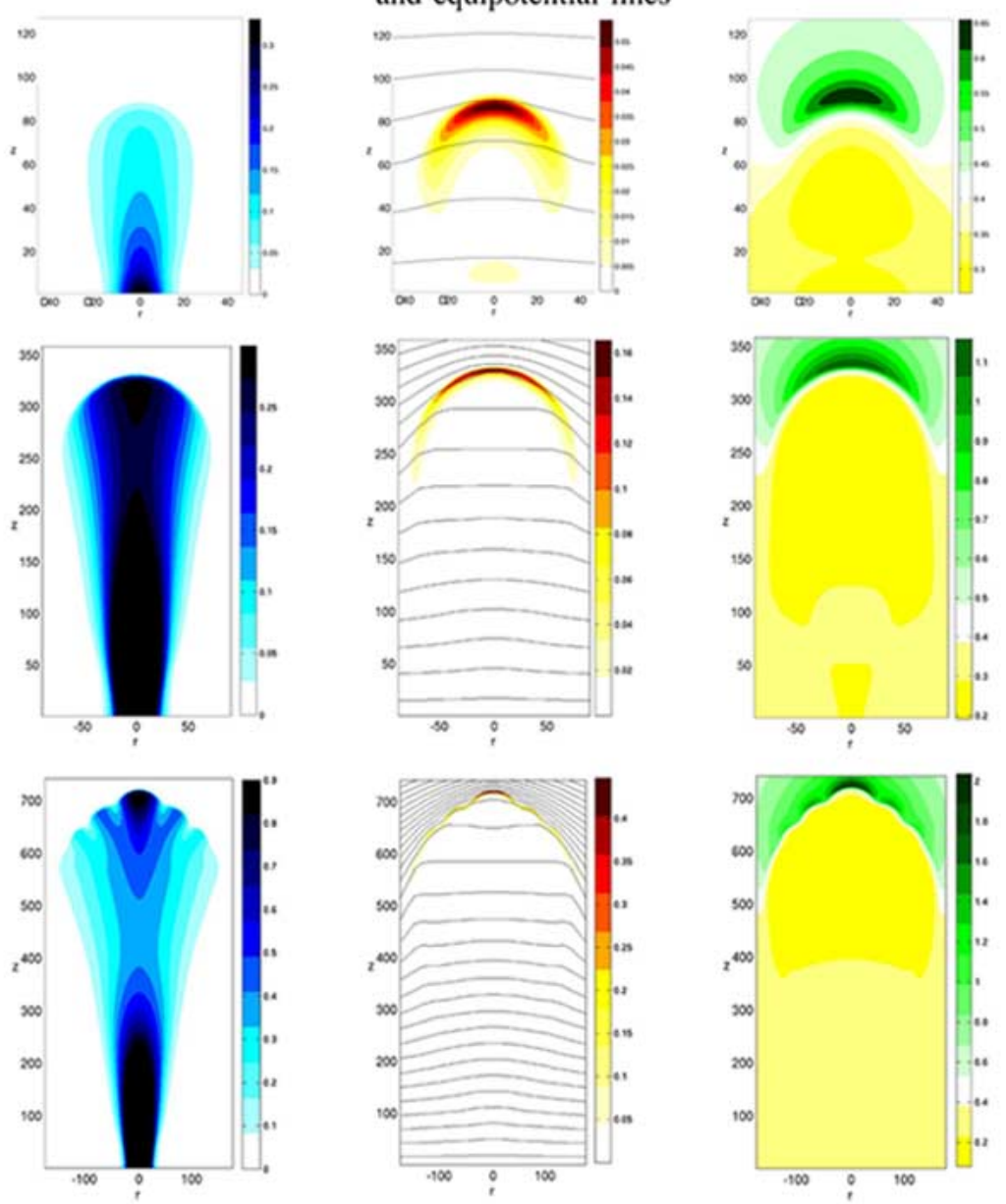

Fig. 1. Temporal evolution of the electron density $\sigma$ (first column), positive ion density $\rho$ (second column), total charge density $\sigma-\rho$ and equipotential lines $\phi$ (third column), and the electric field strength $|\boldsymbol{E}|$ (last column). The background electric field always corresponds to the white level. The rows correspond to $t=100,300,500$, respectively. Results are in dimensionless units and apply to nonattaching gases. For $N_{2}$ at $300 \mathrm{~K}$, the units with their pressure dependence is given by (4).

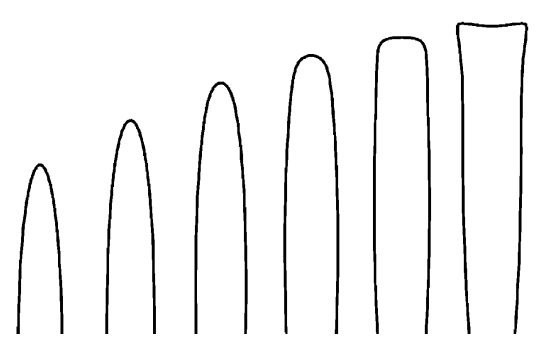

Fig. 2. Temporal evolution of a streamer in the moving boundary approximation. Convex streamer becomes flatter and eventually concave.

The physical nature of the instability is illustrated in Fig. 2. Here, a simplification of the streamer problem is considered as first suggested by Lozansky and Firsov: the interior of the streamer is assumed to be equipotential, the ionization front to be infinitely thin and the interface velocity to be the local electron drift velocity. New analytical solutions of this problem can be found in [8]. Here, we illustrate that this model indeed can exhibit spontaneous branching: the propagating tip becomes flatter and eventually branches. Such an off-axis branching can also be observed in simulations.
The dependence of the branching on macroscopic control parameters is presently under investigation.

\section{REFERENCES}

[1] U. Ebert and W. van Saarloos, "Propagation and structure of planar streamer fronts," Phys. Rev. E, Stat. Phys. Plasmas Fluids Relat. Interdiscip. Top., vol. 55, no. 2, pp. 1530-1549, Feb. 1997.

[2] S. K. Dhali and P. F. Williams, "Two-dimensional studies of streamers in gases," J. Appl. Phys., vol. 62, no. 12, pp. 4696-4707, Dec. 1987.

[3] P. A. Vitello, B. M. Penetrante, and J. N. Bardsley, "Simulation of negative-streamer dynamics in nitrogen," Phys. Rev. E, Stat. Phys. Plasmas Fluids Relat. Interdiscip. Top., vol. 49, no. 6, pp. 5574-5598, Jun. 1994

[4] M. Arrayás, U. Ebert, and W. Hundsdorfer, "Spontaneous branching of anode-directed streamers between planar electrodes," Phys. Rev. Lett., vol. 88, p. 174502 , Apr. 2002.

[5] A. Rocco, U. Ebert, and W. Hundsdorfer, "Branching of negative streamers in free flight," Phys. Rev. E, Stat. Phys. Plasmas Fluids Relat. Interdiscip. Top., vol. 66, p. 035 102(R), Sep. 2002.

[6] N. Liu and V. P. Pasko, "Effects of photoionization on propagation and branching of positive and negative streamers in sprites," J. Geophys. Res., vol. 109, p. A04301, Apr. 2004.

[7] C. Montijn, W. Hundsdorfer, U. Ebert, and J. Wackers, Numerical Simulations of Growing and Branching Ionization Channels Using Local Grid Refinements, submitted for publication.

[8] B. Meulenbroek, A. Rocco, and U. Ebert, "Streamer branching rationalized by conformal mapping techniques," Phys. Rev. E, Stat. Phys. Plasmas Fluids Relat. Interdiscip. Top., vol. 69, p. 067 402, Jun. 2004. 